\title{
Les ruptures et accidents graves de barrages de 1964 à 1983
}

\section{Ruptures and serious accidents on dams from 1964 to 1983}

\author{
Alain Lebreton \\ Ingénieur en chef des Ponts et Chaussées \\ Secrétaire général \\ du Comité technique permanent des barrages
}

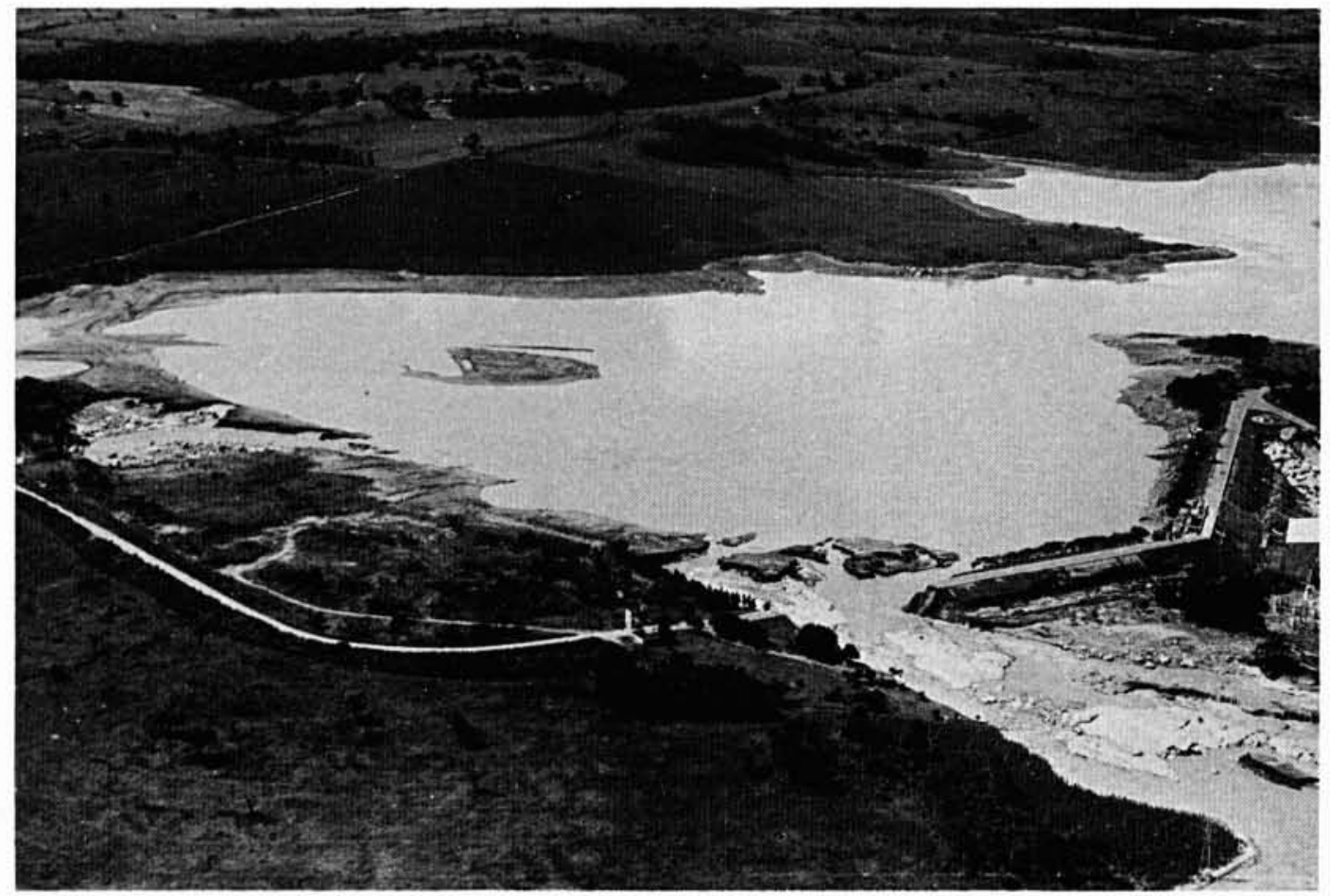

Barrage Armando de Sables Oliveria (Brésil)

Pour ces 20 dernières années, l'étude, objet de cet article. présente un recensement, à peu près exhaustif, des nuptures de grands barrages (hauteur d'au moins $15 \mathrm{~m}$ au-dessus des fondations) et un recensement partiel, à partir d'une ample consultation de la littérature, des ruptures de barrages plus petits. Elle analyse ces accidents : fréquence, causes, répartition géographique et selon l'âge et le type du barrage. Elle examine enfin les conséquences de ces accidents (nombre de victimes) et propose une évaluation des risques pour la population dus à ces ouvrages.
For the last 20 years, the study described in this paper gives an almost complete list of ruptures of major dams (height at least $15 \mathrm{~m}$ above the foundations) and a partial list for smaller dams, obtained from extensive consultation of the literature. It analyzes these accidents: frequency. causes, geographic distribution and distribution according to the age and type of dam. Finally, it examines the consequences of these accidents (number of victims) and proposes an evaluation of the hazards to which the population is exposed due to these dams. 
La nouvelle étude des accidents de barrages qui vous est présentée à plusieurs originalités :

- elle ne porte que sur vingt ans, mais ces vingt années sont les dernières connues (1964-1983);
- elle s'intéresse aux victimes de ces accidents; - elle englobe tous les barrages dont les accidents ont pu être recensés, même si ce ne sont pas des grands barrages ( $15 \mathrm{~m}$ de hauteur) au sens de la Commission Internationale des Grands Barrages (CIGB).

\section{Recensement}

Cette étude a profité d'un dépouillement systématique de revues techniques depuis 1964 et notamment des revues anglo-saxonnes "Engineering News Record" et "Water Power and Dam Construction". Elle a utilisé aussi les résultats des travaux de la CIGB (brochures et rapports des congrès) ainsi que les travaux personnels de MM. Goubet, Schnitter et autres.

Ont été considérés comme accidents de barrages tous ceux ayant conduit à des lâchures involontaires et importantes d'eau à l'aval. Ont été exclus tous les ouvrages qui n'ont pas été conçus comme des barrages définitifs et notamment les digues de stériles, les barrages naturels et les batardeaux de protection de chantier. Ont été inclus, par contre, les accidents, en cours de construction, sur l'ouvrage principal.

Les tableaux $I$ à $I V$ donnent les listes et indications relatives à ces accidents. Les tableaux I et II comportent tous les barrages, dans l'ordre chronologique sur le tableau $I$, dans l'ordre alphabétique des pays et des barrages pour le tableau II (sans les observations, ni références). Les tableaux III et IV, eux aussi par ordre alphabétique des pays et sans observations ni références, donnent séparemment la liste des accidents de barrages de plus et de moins de quinze mètres de haut. Cette liste est, pensons-nous, à peu près complète pour les plus grands barrages, et par contre probablement très incomplètes pour les plus petits.

\section{Analyse des accidents}

\section{Analyse globale}

Les tableaux $V \dot{a} X$ sont des tableaux d'analyse synthétique des accidents recensés. A partir des évaluations du nombre de barrages de plus de $15 \mathrm{~m}$ dans le monde, faites par M.A. Goubet pour l'année 1977, année assez bien située dans la période 1964-1984 étudiée, et des estimations de la proportion de digues dans le total des barrages faites par la CIGB (DBR - 1983), voir tableau XI, des fréquences d'apparition de certains types d'accidents ont été calculés pour barrages de plus de $15 \mathrm{~m}$.

Le nombre moyen d'accidents, pour tous ceux recensés, est de 3,6 par an avec un écart type de 3,2. Pour les seuls barrages d'au moins $15 \mathrm{~m}$ il est environ 2 par an avec un écart-type de 2,6. Si ce nombre suit une loi de Poisson, ce qui n'est pas évident d'après les valeurs constatées, $13 \%$ des années serait sans accident, $26 \%$ avec 1 accident, 27 avec 2,19 avec $3,9,5$ avec 4,4 avec 5 et environ $1,5 \%$ avec plus de 5. Les valeurs observées montrent en fait plus d'années extrêmes (30\% sans accident, $5 \%$ avec plus de 5,11 accidents en 1976), en notant que cette série n'a que vingt valeurs.

On peut toutefois prétendre que les ruptures de barrages en construction, notamment la submersion de digues par des crues, ne sont que des incidents prévisibles et acceptés, les ouvrages de dérivation des eaux n'étant le plus souvent conçus que pour faire face à une crue décennale. Les ruptures de vannes, souvent, ne sont pas non plus considérées comme de graves accidents. Il faut tout de même noter que les ruptures de barrage en construction ont fait 280 morts et les ruptures de vannes 111, au cours de ces 20 ans. Néanmoins, si on les exclut, le nombre moyen d'accidents, pour les barrages en service d'au moins $15 \mathrm{~m}$, hors ruptures de vanne, n'est plus ainsi que de 1,4 par an en moyenne, avec un écart type de 1,9. Si ce nombre d'accidents par an suit une loi de Poisson, avec les mêmes réserves que ci-dessus, il y aurait un quart d'années sans accident ( $35 \%$ constatés), un tiers avec un accident, un quart avec deux accidents, une sur dix avec trois accidents et une sur vingt avec plus de trois.

Pour les barrages en service, d'au moins $15 \mathrm{~m}$, la fréquence des ruptures serait d'environ 1 par 10000 barrages et par an.

\section{Zones géographiques}

Le tableau $V$, par zones géographiques, met une nouvelle fois en évidence des fréquences d'accidents plus élevées aux Etats-Unis que dans le reste du monde et surtout plus élevées qu'en Europe et au Japon. L'analyse par type de barrage explique au moins une partie de ce phénomène.

\section{Construction et mise en eau}

Le tableau VI montre que, pour les nouveaux barrages construits et d'au moins $15 \mathrm{~m}$ de hauteur finale, la fréquence de rupture pendant la construction serait, pour les digues, d'au moins 1,9/1000 digues, et pendant le premier remplissage et les dix premières années, toujours pour les digues, d'environ 1,6/1000. Il n'y a eu qu'un accident de barrage en maçonnerie ou en béton après construction pendant ces vingt années. La fréquence de rupture de ces barrages doit donc être inférieure à celle des digues sans pouvoir être vraiment chiffrée.

\section{Type de barrages}

Le tableau VII montre que la fréquence de ruptures des digues en service est environ quatre fois plus élevée que celle des barrages en béton ou en maçonnerie $\left(1,5.10^{-4}\right.$ contre $\left.0,4 \cdot 10^{-4}\right)$. Ceci explique, au moins en partie, la 
différence constatée sur le tableau V entre USA et Europe, la proportion de digues étant plus élevée aux USA. Le tableau VIII montre, en effet, que les fréquences de rupture pour les digues et autres barrages ne sont pas si différentes selon les diverses zones géographiques.

\section{Age du barrage}

Le tableau IX montre, pour les barrages de plus de $15 \mathrm{~m}$, qu'environ 1/4 des accidents se produit au cours de la construction, $1 / 4$ à la première mise en eau et pendant les 5 premières années et la moitié en service. Cette dernière proportion est beaucoup plus élevée $(87 \%)$ pour les barrages de moins de $15 \mathrm{~m}$, mais cela résulte probablement d'un biais de l'échantillon, la submersion de petites digues en construction ne faisant pratiquement jamais l'objet d'articles de presse en raison du peu d'importance des conséquences.

\section{Causes de rupture des digues}

Le tableau $X$ analyse les causes de ruptures des digues; pour les digues d'au moins $15 \mathrm{~m}$, la moitié des ruptures sont causées par des crues (submersion ou érosion par l'aval) et l'autre moitié par des renards ou glissements. Pour les digues de moins de $15 \mathrm{~m}$, cette proportion est plutôt de $3 / 4,1 / 4$.

\section{Causes des ruptures des barrages en béton ou maçon- neries}

Les causes sont indiquées dans les renvois du tableau VII. Il y a eu 5 ruptures, dont 2 sont survenues lors de crues (submersion et érosion par l'aval), 2 par rupture de la structure (renversement et rupture d'un contrefort, et la dernière par une rupture de fondation. Aucune n'est survenue pendant la construction. Le barrage à contrefort était ancien ( 57 ans). Le barrage poids renversé n'avait que 6 ans. Seuls 2 des 5 barrages rompus avaient plus de $15 \mathrm{~m}$ (une à voûte multiple avec rupture des fondations au bout de 13 ans, le barrage poids renversé précité).

\section{Ruptures de vannes}

Bien que non considérées généralement comme de grands accidents de barrages, les 4 ruptures de vannes recensées ont néanmoins fait un nombre important de victimes (111).

\section{Nombre de victimes}

\section{Recensement}

Le tableau XII décompte le nombre de ruptures ayant fait des victimes dont le nombre est connu ou a pu être estimé. Les accidents les plus graves se sont produits aux Indes et en Indonésie, pays évidemment très peuplés. Le nombre total de victimes serait ainsi de 2600 , soit environ 130 par an et 36 par rupture. Tous les accidents graves ont été provoqués, pendant ces 20 ans, par des ruptures de digues, mais il n'en a pas été de même pour les décennies précédentes où de nombreux barrages poids, un barrage voûte (Malpasset), ainsi que l'éboulement dans la retenue de Longaronne, ont fait aussi de nombreuses victimes. Aux USA, le nombre de morts aurait été de 61 (3 par an et moins de 2, en moyenne, par rupture) et en Europe de 71 (un peu plus de 3 par an et 5 , en moyenne, par ruptures recensées). Pour l'ensemble Asie-Afrique-Amérique du Sud, il serait d'environ 2500 (plus de 120 par an et plus de 130, en moyenne, par rupture) mais le seul accident de Macchuu II aux Indes a déjà fait environ 2000 victimes.

\section{Nombre de victimes par rupture}

L'annexe I est une tentative d'analyse plus approfondie du nombre de victimes constaté par rupture. Environ la moitié des accidents ne ferait aucune victime (si l'on suppose qu'il n'y a pas eu de victimes lorsqu'il n'en a pas été fait état). L'ajustement conduit à estimer que, dans un cas sur 10, la rupture ferait plus de 60 victimes environ, et dans un cas sur 100 plus de 800 environ. Les valeurs sont toutefois à prendre avec précautions en raison du faible échantillonnage.

Nous avons donc été conduit à étendre cette étude aux accidents dont le nombre de victimes a pu être retrouvé et s'étant produits depuis l'année 1800, éléments qui avaient été recensés dans une étude non publiée sur la détermination d'un critère simple du danger potentiel des barrages. Les tableaux XIII et XIV en donnent quelques résultats et l'annexe $I I$, sous la même forme que l'annexe $\mathrm{I}$, les analyse. Les ajustements sont meilleurs que ceux de l'annexe I et les nombres calculés sont portés sur la figure 3. On retrouve environ une moitié d'accidents sans victimes, mais des valeurs plus élevées pour les fréquences faibles (240 victimes dans plus de $10 \%$ des cas, 2500 dans plus de 1 cas sur 100). Sur la figure 4 , les courbes de cumul décroissantes sont comparées aux indications données par Schnitter dans la figure 5 de son article de 1976).

Il paraît assez normal que la gravité moyenne des accidents ait diminué depuis 1800; l'amélioration de la surveillance et des communications (téléphone), permettent des alertes plus efficaces: l'amélioration des caractéristiques des barrages poids, de profil souvent insuffisant autrefois, diminue le risque de rupture brutale des ouvrages de ce type.

\section{Nombre de morts par an}

L'analyse du nombre de morts par an (par intervalles de 5 ans et de 10 ans) depuis 1855 et qui est donnée sur le tableau XIV donne pourtant une impression contraire. Avec une extrême variabilité (de 0 à près de 3000 sur les décennies), le nombre moyen de morts par an, supérieur à 100 sur toute la période, paraît avoir évolué d'environ 60 par an vers 1850 à 150 par an actuellement. La baisse de la gravité moyenne par accident semble donc avoir été plus que compensée par l'augmentation du nombre de barrages et du nombre des accidents, ainsi que par 
l'occurence de quelques accidents très graves ayant fait plus de 1000 morts, entre 1960 et 1965 et 1975 et 1980.

\section{Nombre de morts par type de barrages}

Pour la période 1800-1983, le tableau XIII montre que les accidents dus aux ruptures de barrages en béton ou maçonné seraient deux fois plus graves (en moyenne) que ceux de digues en terre ou en enrochements. Ceci paraît confirmé par l'examen de la figure 5. Toutefois cette différence s'estompe pour ce qui concerne les accidents ayant fait le plus de victimes et la $2^{\mathrm{c}}$ partie de l'annexe II ne montre, pour ces accidents, qu'une augmentation d'un tiers environ. Ce phénomène provient sans doute essentiellement du mode de rupture, brutal pour les premiers, progressifs pour les seconds, qui modifie totalement les possibilités d'alerte et d'évacuation des populations. En outre, beaucoup de ruptures de digues surviennent à la suite de crues ayant conduit à un départ des populations concernées hors des zones atteintes par l'onde de rupture.

\section{Conclusion}

En supposant, avec une certaine audace, que les fréquences et écarts observés ces 20 dernières années se retrouveront les 200 prochaines on peut estimer les risques dus aux grands barrages, au sens de la CIGB, c'est-à-dire d'au moins $15 \mathrm{~m}$ de hauteur au-dessus des fondations.

En France, par exemple, il y aura pendant cette période, environ 500 barrages en service (1/3 de digues et $2 / 3$ de barrages en béton ou en maçonnerie) comme le montre le tableau $X V$ et il s'en construira une centaine pendant ces 20 années.

Il devrait se produire en France, au cours de ces 20 prochaines années, un accident de barrage. Plus précisément, si l'on suppose que ce nombre de rupture suivra une loi de Poisson, il y aura $37 \%$ de chances qu'il n'y en ait pas, $37 \%$ de chances qu'il n'y en ait qu'un, $18 \%$ de chances qu'il y en ait deux, $6 \%$ trois, et environ 1 à $2 \%$ qu'il y en ait plus de trois.

En combinant la loi de Poisson de ce nombre d'accidents et les lois (annexe I) approchant le nombre des victimes par accident, on peut estimer le nombre de victimes qui pourrait résulter de ces accidents "statistiques" en France durant ces 20 prochaines années.

Il y a heureusement presque $75 \%$ de chances qu'il n'y ait pas de victimes (notamment dans les cas où il n'y aurait pas d'accidents). Il y a $80 \%$ de chances qu'il y ait moins de 7 morts, $90 \%$ moins de $90,95 \%$ moins de 250 . Il n'y aurait qu'une chance sur 100 que ce nombre, malheureusement, dépasse 900 et une sur mille qu'il dépasse 4000 .

On peut penser que les événements à venir n'ont aucune raison de suivre ces lois statistiques, diverses, approximatives et basées sur le passé. Elles portent de plus sur des événements mondiaux alors qu'il apparait que les risques sont moins élevés dans les pays de vieille civilisation (Japon - Europe). De toute façon, dans notre pays, toutes les dispositions nécessaires sont prises, notamment depuis l'accident de Malpasset en 1959 (421 morts), continueront à être prises et seront, s'il le faut, renforcées en matière d'examen des projets, de surveillance et de contrôle de la réalisation et de l'exploitation, d'entretien, de réparations et de confortements. Il est donc permis d'espérer que l'accident statistique de ces 20 prochaines années ne se produira pas. S'il se produisait, grâce aux plans d'alerte qui ont été mis en place ou qui vont l'être, il devrait faire partie de ceux qui ne font pas, ou très peu de victimes.

Nota: Cette étude n'a pas pris en compte les risques dus aux ruptures de dépôts de stériles miniers saturés d'eau. Les coulées de boue qui en résultent ensevelissent tout et peuvent être plus meurtrières que les lâchures d'eau résultant de ruptures de barrages de taille analogue. La démonstration vient encore d'en être faite récemment à Tesero en Italie (214 morts). Il en avait été de même en Virginie aux USA en 1972 (125 morts) à Aberfan au Pays de Galles en 1966 (144 morts) et en 1965 à El Cobre au Chili (200 morts). En outre, les précautions prises pour assurer la sécurité ne sont probablement pas les mêmes que pour les barrages.

\section{Références bibliographiques}

(avec les abréviations utilisées).

[1] ENR : Engineering News Record.

[2] WP : Water Power and Dam Construction.

[3] HB : La Houille Blanche, A. GouBET, Risques associés aux barrages n० 8/1979.

[4] CETEGREF : Centre technique du génie rural, des eaux et des forêts, à Antony (France).

[5] COIMBRA : Rapports à la Conférence internationale sur la sécurité des barrages, Coimbra 23-28 avril 1984.

[6] USCOLD-DSRC : USCOLD, avril 1972, Dam Safety Practices and Concerns in the United States.
[7] USDI-DPS : US Department of the Interior, Dams and Public Safety, 1980.

[8] CIGB Qxx: Congrès international des grands barrages, Compte-rendu de la Question xx (notamment Q 45 et Q 49).

[9] CIGB-LFDI : CIGB, Lessons from dam incidents, 1974.

[10] USCOLD-LFDI : ASCE et USCOLD, Lessons from dam incidents (USA), 1975.

[11] CIGB : Répertoire des Grands Barrages - 1973, 1975, 1977.

[12] CIGB-DBR : Détérioration des barrages et réservoirs, recueil de cas et analyse, décembre 1983.

[13] SCHNITTER : WASSER, Energie, Luft - Eau, énergie, air no 5, 1976 - Sûreté statistique des barrages. 


\section{Tableaux} et annexes

Tableau I. - Ruptures de barrages (1964-1983) (sauf barrages naturels, dépôts de stériles, batardeaux de chantier.

Tableau II. - Ruptures de barrages (1964-1983).

Tableau III. - Ruptures de barrages d'au moins 15 mètres (1964-1983).

Tableau IV. - Ruptures de barrages de moins de 15 mètres (1964-1983).

Tableau V. - Ruptures de barrages par zones géograhiques (1964-1983).

Tableau VI. - Ruptures de barrages d'au moins $15 \mathrm{~m}$ (hauteur finale) pendant leur construction et leurs premières années (1964-1983).

Tableau VII. - Ruptures de barrages par types d'ouvrages (1964-1983).

Tableau VIII. - Ruptures de barrages en service d'au moins $15 \mathrm{~m}$ par zones géographiques et par types d'ouvrage.

Tableau IX. - Age des barrages rompus (1964-1983).

Tableau X. - Digues - Causes des ruptures (1964-1983) - sauf ruptures de vannes.

Tableau XI. - Estimation du nombre de barrages en service d'au moins $15 \mathrm{~m}$ dans le monde (en 1977).

Tableau XII. - Nombre de ruptures ayant fait des victimes connues et nombre de victimes connues (1964-1983).

Tableau XIII. - Nombre de ruptures $(\mathrm{Nb})$ ayant fait des victimes connues et nombre de victimes $(\mathrm{N})$ selon le type de rupture et par pays (1800-1983).

Tableau XIV. - Évolution du nombre de victimes d'accidents de barrages au cours des années depuis 1855.

Tableau XV. - Barrages en service en France, selon la définition CIGB (répertoire à jour en 1982).

Annexe I. - Distribution des accidents selon le nombre des victimes (1964-1983).

Annexe II. - Distribution des accidents selon le nombre des victimes (1800-1983). 


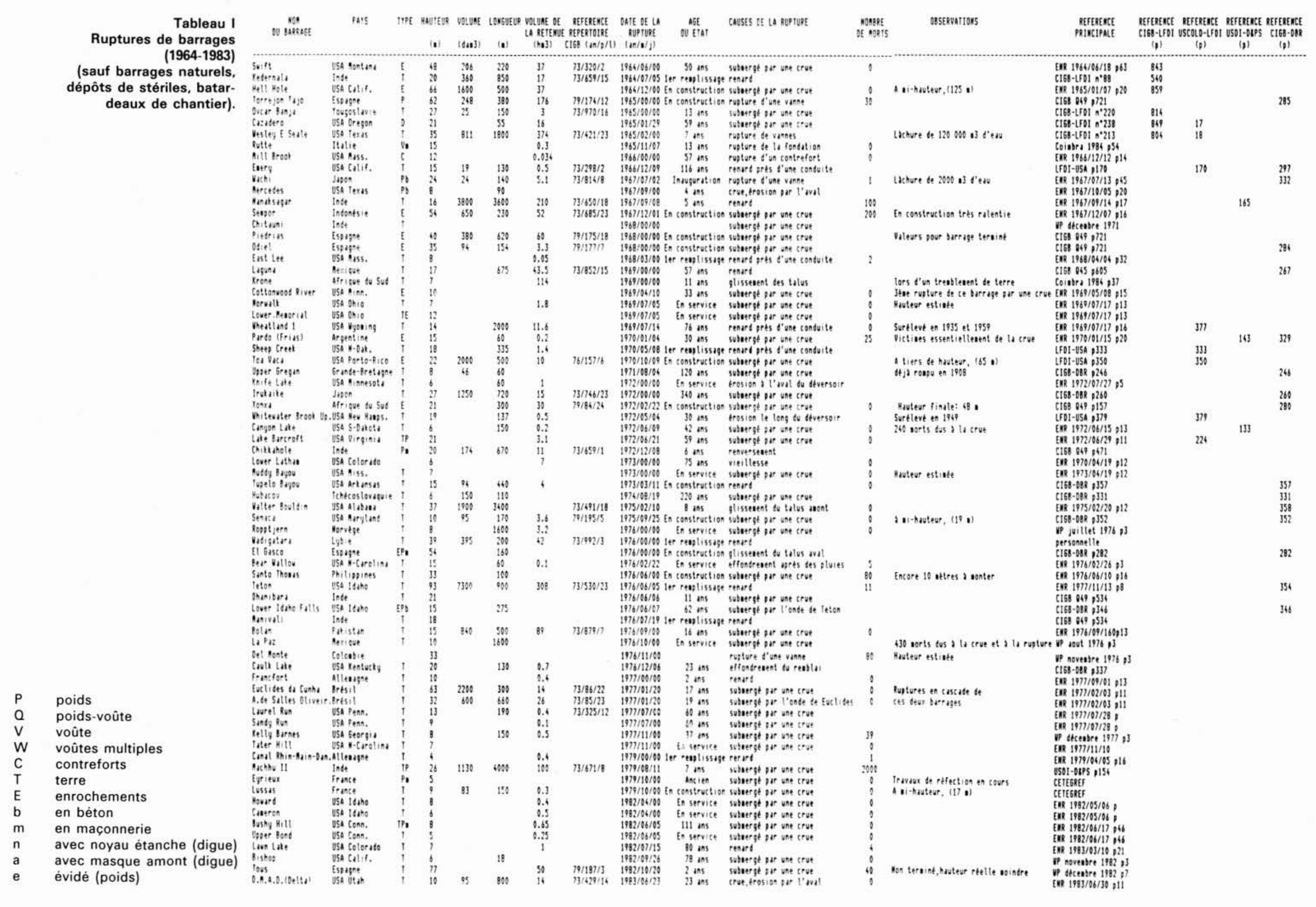




\begin{tabular}{|c|c|c|}
\hline $\begin{array}{l}\text { Mor } \\
\text { OU Barrage }\end{array}$ & Pars & 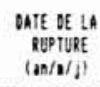 \\
\hline Krone & Afrique du Sud & $1969 / 00 / 00$ \\
\hline $\operatorname{lonxa}$ & Afrique du Sud & $1972 / 102 / 22$ \\
\hline Canal Rhin-hain & n.Allesagne & $1979 / 00 / 00$ \\
\hline 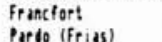 & Alleasinge & 1977700000 \\
\hline $\begin{array}{l}\text { iarto Ifrias) } \\
\text { a.ce Salles Olive i. }\end{array}$ & $\begin{array}{l}\text { Argentine } \\
\text { Bretsit }\end{array}$ & $\begin{array}{l}1990 / 101 / 144 \\
197 / 01 / 20\end{array}$ \\
\hline Euclides at cunha & Bressil & $1977 / 01 / 20$ \\
\hline Del Aonte & Colonbie & $1976 / 11100$ \\
\hline El Gasco & Espogne & $1976 / 00 / 00$ \\
\hline 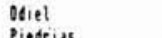 & Espogne & $1968 / 00 / 00$ \\
\hline $\begin{array}{l}\text { Pietrias } \\
\text { Torrejon Tajo }\end{array}$ & $\begin{array}{l}\text { Eslagne } \\
\text { Espigne }\end{array}$ & $\begin{array}{l}1988 / 10 / 000 \\
1965 / 00 / 00\end{array}$ \\
\hline & $\begin{array}{l}\text { Espagne } \\
\text { Esper }\end{array}$ & $1982 / 10 / 20$ \\
\hline Eyrieux & Frombe & $1979 / 10 / 00$ \\
\hline & France & $1979 / 10 / 00$ \\
\hline Upper Gregan & Grande-Bretagne & e 1971/08/04 \\
\hline 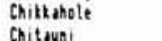 & Inde & $1972 / 12 / 08$ \\
\hline 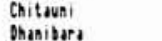 & Inde & $1968 / 00 / 00$ \\
\hline 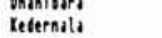 & $\begin{array}{l}\text { Inde } \\
\text { inde }\end{array}$ & $\begin{array}{l}1976 / 066 / 06 \\
1964601705\end{array}$ \\
\hline nachnu If & $\begin{array}{l}\text { Inte } \\
\text { Inde }\end{array}$ & $1979 / 108 / 11$ \\
\hline Aenivati & Inde & $1976 / 07 / 19$ \\
\hline Menatsugar & Inde & $1967 / 09 / 08$ \\
\hline Seapor & Indontsie & $1967 / 1270 !$ \\
\hline $\begin{array}{l}\text { latte } \\
\text { Irutaits }\end{array}$ & Inlie & $1965 / 11 / 07$ \\
\hline $\begin{array}{l}\text { Wachi } \\
\text { Wut }\end{array}$ & $\begin{array}{l}\text { Japon } \\
\text { Japon }\end{array}$ & $\begin{array}{l}1977 / 270 / 0 / 00 \\
196 / / 02 / 102\end{array}$ \\
\hline Vadigatara & Lybie & $1976 / 00 / 100$ \\
\hline & Rexique & $1976 / 10 / 00$ \\
\hline Laguna & Rexique & $1969 / 00 / 00$ \\
\hline Ropitjeen & $\begin{array}{l}\text { Morvege } \\
\text { petgen }\end{array}$ & $1976 / 00 / 00$ \\
\hline $\begin{array}{l}\text { bolan Thous } \\
\text { Sunto The }\end{array}$ & $\begin{array}{l}\text { Patistan } \\
\text { Philizenges. }\end{array}$ & $\begin{array}{l}1976 / 109 / 00 \\
1996010600\end{array}$ \\
\hline Hobcove & Therecosloviavie & $\begin{array}{l}17 / 601 / 086100 \\
\end{array}$ \\
\hline Bear Villov & USA N-Carolina & $1976 / 02 / 22$ \\
\hline Bishop & USA Calif. & $1982 / 09 / 26$ \\
\hline lushy Hill & USA Conn. & $1982 / 06 / 105$ \\
\hline Canteron & USA I tatho & $1982 / 106 / 100$ \\
\hline $\begin{array}{l}\text { acagon Late } \\
\text { Cowlk Late }\end{array}$ & 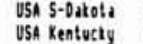 & $\begin{array}{l}1972 / 106 / 199 \\
1976 / 1 / 106\end{array}$ \\
\hline Candero & $\begin{array}{l}\text { USA Dregon } \\
\text { USA }\end{array}$ & $1965 / 01 / 29$ \\
\hline Cottonvood River & usa ninn. & $1969 / 06 / 10$ \\
\hline D.A.A.D.Delta) & USA Utah & $1983 / 06 / 23$ \\
\hline East lee & USA Rass: & $1968 / 03 / 00$ \\
\hline & USA Calif. & $1966 / 12 / 09$ \\
\hline $\begin{array}{l}\text { Hell Hole } \\
\text { Hower }\end{array}$ & USA calif. & $1966 / 112 / 00$ \\
\hline $\begin{array}{l}\text { Relly barnes } \\
\text { Kello }\end{array}$ & $\begin{array}{l}\text { USA I dohno } \\
\text { USA Georgia }\end{array}$ & $\begin{array}{l}1982870 / 4 / 100 \\
197 / 11 / 00\end{array}$ \\
\hline Inife latt & USA Ainnesoti & $1972 / 100 / 100$ \\
\hline Late Burcrooft & USA Virginid & 1972/06/21 \\
\hline Larrel Run & USA Penn & $19777107 / 100$ \\
\hline solk & & \\
\hline & USA 16 & $1976 / 06 / 07$ \\
\hline Lover Latha & USA Colorido & $1973 / 00 / 00$ \\
\hline $\begin{array}{l}\text { Lover Atearial } \\
\text { Aercotes }\end{array}$ & $\begin{array}{l}\text { USA Ohio } \\
\text { SISA }\end{array}$ & $1969 / 07 / 105$ \\
\hline $\begin{array}{l}\text { Merceteses } \\
\text { Mill leook }\end{array}$ & 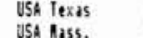 & $1967 / 109 / 100$ \\
\hline $\begin{array}{l}\text { Mill broot } \\
\text { Avedy Bayyo: }\end{array}$ & $\begin{array}{l}\text { USA Mass. } \\
\text { USA Riss. }\end{array}$ & $\begin{array}{l}1966 / 00000 \\
1973 / 00100\end{array}$ \\
\hline & USA Ohio & $\begin{array}{l}1973 / 01010700 \\
196907 / 05\end{array}$ \\
\hline Sunty Run & USA Pe & 1977 \\
\hline Sentia & USA Aaryland & $1975 / 09 / 25$ \\
\hline $\begin{array}{l}\text { Sheep Greet } \\
\text { Svift }\end{array}$ & USA H-Dit. & $1970 / 05$ \\
\hline & USA Montzan & $1964 / 106$. \\
\hline & 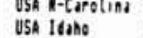 & $\begin{array}{l}199771111100 \\
197660605\end{array}$ \\
\hline Tos Viet & USA Porto-Rise & $1970 / 10 / 09$ \\
\hline & USA AR & $1973 / 0$ \\
\hline & cois & $1982 / 06 / 05$ \\
\hline 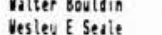 & 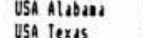 & $1975 / 02 / 10$ \\
\hline $\begin{array}{l}\text { essleyt E sealt } \\
\text { theatind! }\end{array}$ & $\begin{array}{l}\text { USA exas } \\
\text { usa Wyaning }\end{array}$ & $\begin{array}{l}19655902700 \\
196907 / 16\end{array}$ \\
\hline & USA Meu Haoss. & $1972 / 05 / 04$ \\
\hline & Youyostavie & $1965 / 00 / 00$ \\
\hline
\end{tabular}

Tableau III

Ruptures de barrages au moins 15 mètre (1964-1983).

\begin{tabular}{|c|c|c|}
\hline $\begin{array}{l}\text { ROR } \\
\text { OU BARRAGE }\end{array}$ & pars & $\begin{array}{l}\text { DATE OE LA } \\
\text { RUPTURE } \\
\text { (mon/s/d) }\end{array}$ \\
\hline & $\begin{array}{l}\text { Aifrique du Sud } \\
\text { Argentine }\end{array}$ & $\begin{array}{l}1972 / 02 / 22 \\
1970 / 01 / 104\end{array}$ \\
\hline & bresil & $19771 / 11 / 20$ \\
\hline "W Cunha & Eitsit! & $\begin{array}{l}19771 / 101 / 20 \\
1976 / 11 / 00\end{array}$ \\
\hline & $\begin{array}{l}\text { Elolobile } \\
\text { Espignte }\end{array}$ & $\begin{array}{l}1 / 11 / 100 \\
10000\end{array}$ \\
\hline & & \\
\hline & & \\
\hline & & \\
\hline & & \\
\hline & $\begin{array}{l}\text { Inde } \\
\text { inde }\end{array}$ & $1976 / 06 / 1$ \\
\hline & & \\
\hline & & \\
\hline & & \\
\hline & & \\
\hline & & \\
\hline & & \\
\hline & & \\
\hline & & \\
\hline & & \\
\hline & & \\
\hline 25 & & \\
\hline & 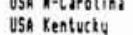 & 1976 \\
\hline & & \\
\hline & & \\
\hline & & \\
\hline & U5A & \\
\hline 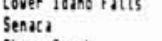 & $\begin{array}{l}\text { USA } \\
\text { USA A }\end{array}$ & 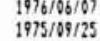 \\
\hline & & \\
\hline & & \\
\hline & & \\
\hline & & \\
\hline utsin & & $\begin{array}{l}1973 / 1 / 7311 \\
1995 / 02 / 1\end{array}$ \\
\hline & USA Texis & \\
\hline & & \\
\hline & Tougoslavie & $1965 / 00 / 00$ \\
\hline
\end{tabular}

thour les burrages en construction.
Tableau IV

Ruptures de barrages de moins de 15 mètres (1964-1983).

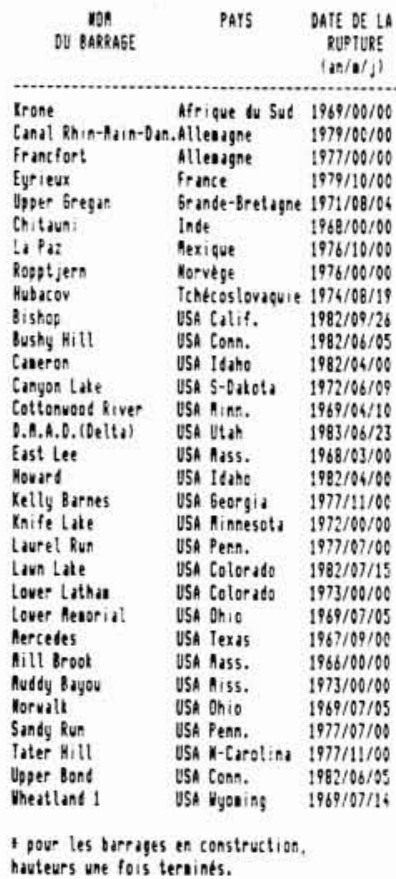


Tableau V. - Ruptures de barrages par zones géograhiques (1964-1983).

\begin{tabular}{|c|c|c|c|c|c|c|c|}
\hline \multirow[t]{2}{*}{ Zones géographiques } & \multicolumn{5}{|c|}{ Nombre de ruptures } & \multicolumn{2}{|c|}{ Fréquence de ruptures $(H \geqslant 15 \mathrm{~m})$} \\
\hline & Total & $\mathrm{H}<15 \mathrm{~m}$ & в $\geqslant 15 \mathrm{~m}$ & $\begin{array}{l}H \geqslant 15 \mathrm{~m} \\
\text { sauf ruptures } \\
\text { de vannes }\end{array}$ & $\begin{array}{l}\mathrm{H} \geqslant 15 \mathrm{~m} \\
\text { sauf ruptures } \\
\text { de vannies et } \\
\text { barrages en } \\
\text { construction }\end{array}$ & $\begin{array}{l}\text { Toutes ruptures } \\
\text { (nombre de rupture } \\
\text { an) }\end{array}$ & $\begin{array}{l}\text { sauf barrages en construction } \\
\text { et ruptures de vannes } \\
\text { par nombre de barrages (1) et par }\end{array}$ \\
\hline USA & 38 & 22 & 16 & 15 & 11 & $2,2 \cdot 10^{-4}$ & $1,5 \cdot 10^{-4}$ \\
\hline EUROPE & 14 & 6 & 8 & 7 & 3 & $1,6.10^{-4}$ & $0,6 \cdot 10^{-4}$ \\
\hline AMERIQUE (sauf USA) & 6 & 1 & 5 & 4 & 4 & & \\
\hline ASIE & 12 & - & 12 & 11 & 9 & $\left\{\begin{array}{l}1,5 \cdot 10^{-4} \\
\left(0,5 \cdot 10^{-4} \text { au Japon }\right)\end{array}\right.$ & $\begin{array}{l}1,1 \cdot 10^{-4} \\
\left(0,25 \cdot 10^{-4} \text { au Japon }\right)\end{array}$ \\
\hline AFRIQUE & 3 & 1 & 2 & 2 & 1 & & \\
\hline TOTAL & 73 & 30 & 43 & 39 & 28 & $1,7^{10-4}$ & $1,1.10^{-4}$ \\
\hline
\end{tabular}

(1) Nombre de barrages évalué à partir de H.B. A.GOUBET p.476 (Nb de barrages de plus de 15 m en 1977)

Tableau VI. - Ruptures de barrages d'au moins 15 m (hauteur finale) pendant leur construction et leurs premières années (1964-1983).

\begin{tabular}{|c|c|c|c|c|c|c|c|}
\hline \multirow[t]{2}{*}{ Etat ou âge } & \multicolumn{4}{|c|}{ Nombre de ruptures } & \multicolumn{3}{|c|}{$\begin{array}{l}\text { Fréquence des ruptures } \\
\text { (nombre par barrages construits (1)) }\end{array}$} \\
\hline & Digues & Autres & $\begin{array}{l}\text { Ruptures } \\
\text { de vannes }\end{array}$ & Total & Digues & Autres & Total \\
\hline En construction & $11^{(2)}$ & - & 1 & 12 & $1,9 / 1000$ & - & $1,7 / 1000$ \\
\hline $\begin{array}{l}\text { Au ler remplissage } \\
\text { et pendant les } \\
5 \text { premières années }\end{array}$ & $7^{(3)}$ & - & 1 & 8 & $1,2 / 1000$ & - & $1,1 / 1000$ \\
\hline $\begin{array}{l}\text { Au ler remplissage } \\
\text { et pendant les } \\
10 \text { premiềres années }\end{array}$ & $9^{(4)}$ & $1^{(5)}$ & 2 & 12 & $1,6 / 1000$ & $0,8 / 1000$ & $1,7 / 1000$ \\
\hline
\end{tabular}

(1) Le nombre de barrage construit en 20 ans est évalué à 7000 (H.B. A.GOUBET p. 477) dont 5800 digues ( 5 sur 6 d'après CIGB-DBR).

(2) 9 ruptures par submersion lors de crues et bréches, une par renard.

(3) 6 ruptures par renard et une par submersion de crue.

(4) de 6 à 10 ans : 1 rupture par submersion de crue et une par glissement de talus de la digue.

(5) Renversement d'un barrage-poids. 
Tableau VII. - Ruptures de barrages par types d'ouvrages (1964-1983).

\begin{tabular}{|c|c|c|c|c|c|c|c|}
\hline \multirow[t]{2}{*}{ Types d'ouvrages } & \multicolumn{4}{|c|}{ Nombre de ruptures } & \multirow{2}{*}{$\begin{array}{l}\text { Evaluation du nombre } \\
\text { de barrages d'au } \\
\text { moins } 15 \mathrm{~m} \\
\text { (en 1977) }\end{array}$} & \multirow{2}{*}{$\begin{array}{l}\text { Fréquences de ruptures } \\
\text { (Nombre de ruptures par } \\
\text { Toutes ruptures }\end{array}$} & \multirow{2}{*}{$\begin{array}{l}(H \geqslant 15 \mathrm{~m}) * \\
\text { barrage et par an) } \\
\text { sauf barrages en } \\
\text { construction }\end{array}$} \\
\hline & \begin{tabular}{|l|} 
Tous \\
barrages
\end{tabular} & $\begin{array}{c}\mathrm{H}<15 \mathrm{~m} \\
*\end{array}$ & $\begin{array}{c}H \geqslant 15 \mathrm{~m} \\
*\end{array}$ & $\begin{array}{l}H \geqslant 15 \mathrm{~m} \\
\text { sauf en } \\
\text { construc- } \\
\text { tion }\end{array}$ & & & \\
\hline Digues en terre & 52 & 26 & 26 & 22 & & $2,10^{-4}$ & $=0^{-4}$ \\
\hline $\begin{array}{l}\text { Digues en enroche- } \\
\text { ments }\end{array}$ & 11 & 1 & 10 & $3^{(1)}$ & 8500 & 2,100 & 10 \\
\hline Barrages-poids & 3 & $2^{(2)}$ & $1^{(3)}$ & 1 & & & \\
\hline $\begin{array}{l}\text { Barrages à contre- } \\
\text { forts et voûtes } \\
\text { multiples }\end{array}$ & 2 & $1^{(4)}$ & $1^{(5)}$ & 1 & 4200 & $0,4 \cdot 10^{-4}$ & $0,4 \cdot 10^{-4}$ \\
\hline Divers & 1 & - & $1^{(6)}$ & 1 & ) & & \\
\hline Ruptures de vannes & 4 & - & 4 & 3 & & & \\
\hline TOTAL & 73 & 30 & 43 & 31 & 12700 & $1,7 \cdot 10^{-4}$ & $1,2.10^{-4}$ \\
\hline
\end{tabular}

(1) 2 submersions par des crues et une par 1 'onde de rupture d'un barrage amont.

(2) I submersion par des crues et une érosion aval par une crue.

(3) renversement.

(4) rupture d'un contrefort.

(5) rupture de la fondation sous le barrage.

(6) barrage en bois et rocher submergé par une crue.

* pour les barrages en construction, hauteur une fois terminés

Tableau VIII. - Ruptures de barrages en service d'au moins 15 m par zones géographiques et par types d'ouvrage.

(barrages rompus par onde amont exclus)

\begin{tabular}{|c|c|c|c|c|c|c|c|c|c|}
\hline \multirow[t]{2}{*}{ Zones géographiques } & \multicolumn{5}{|c|}{ Nombre de ruptures } & \multicolumn{4}{|c|}{ Fréquence des ruptures } \\
\hline & Digues & $\begin{array}{l}\text { Barrage en } \\
\text { béton ou ma- } \\
\text { connerie }\end{array}$ & $\begin{array}{c}\text { Barrages } \\
\text { divers }\end{array}$ & $\begin{array}{l}\text { Ruptures } \\
\text { de } \\
\text { vannes }\end{array}$ & Total & Digues & $\begin{array}{l}\text { Barrage en } \\
\text { béton ou ma- } \\
\text { çonnerie }\end{array}$ & $\begin{array}{l}\text { Barrages } \\
\text { divers }\end{array}$ & Total \\
\hline USA & 9 & 0 & 1 & 1 & 11 & $1,7.10^{-4}$ & 0 & $1,7 \cdot 10^{-4}$ & $1,5 \cdot 10^{-4}$ \\
\hline EUROPE & 2 & 1 & - & - & 3 & $1,3.10^{-4}$ & $0,3 \cdot 10^{-4}$ & - & $0,6 \cdot 10^{-4}$ \\
\hline AMERIQUE (sauf USA) & 3 & - & - & 1 & 4 & 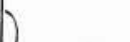 & & & \\
\hline ASIE & 8 & 1 & - & 1 & 10 & $1,6 \cdot 10^{-4}$ & $0,8 \cdot 10^{-4}$ & - & $1,7 \cdot 10^{-4}$ \\
\hline AFRIQUE & 1 & - & - & - & 1 & & & & \\
\hline (JAPON) & 1 & & & 1 & 2 & $0,4 \cdot 10^{-4}$ & 0 & - & $0,5 \cdot 10^{-4}$ \\
\hline TOTAL & 23 & 2 & 1 & 3 & 29 & $1,4 \cdot 10^{-4}$ & $0,3 \cdot 10^{-4}$ & $1,7.10^{-4}$ & $1,1 \cdot 10^{-4}$ \\
\hline
\end{tabular}


Tableau IX. - Age des barrages rompus (1964-1983).

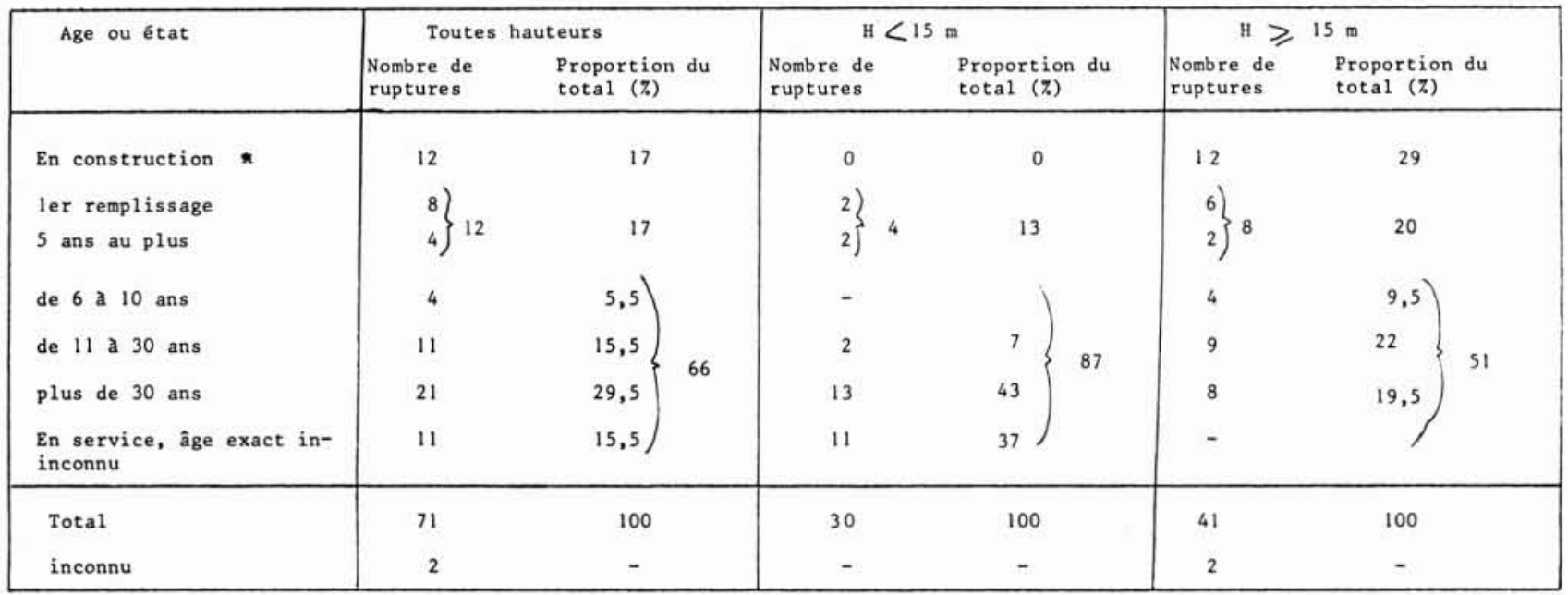

* pour les barrages en construction, hauteur une fois terminés.

Tableau X. - Digues - Causes des ruptures (1964-1983) - sauf ruptures de vannes.

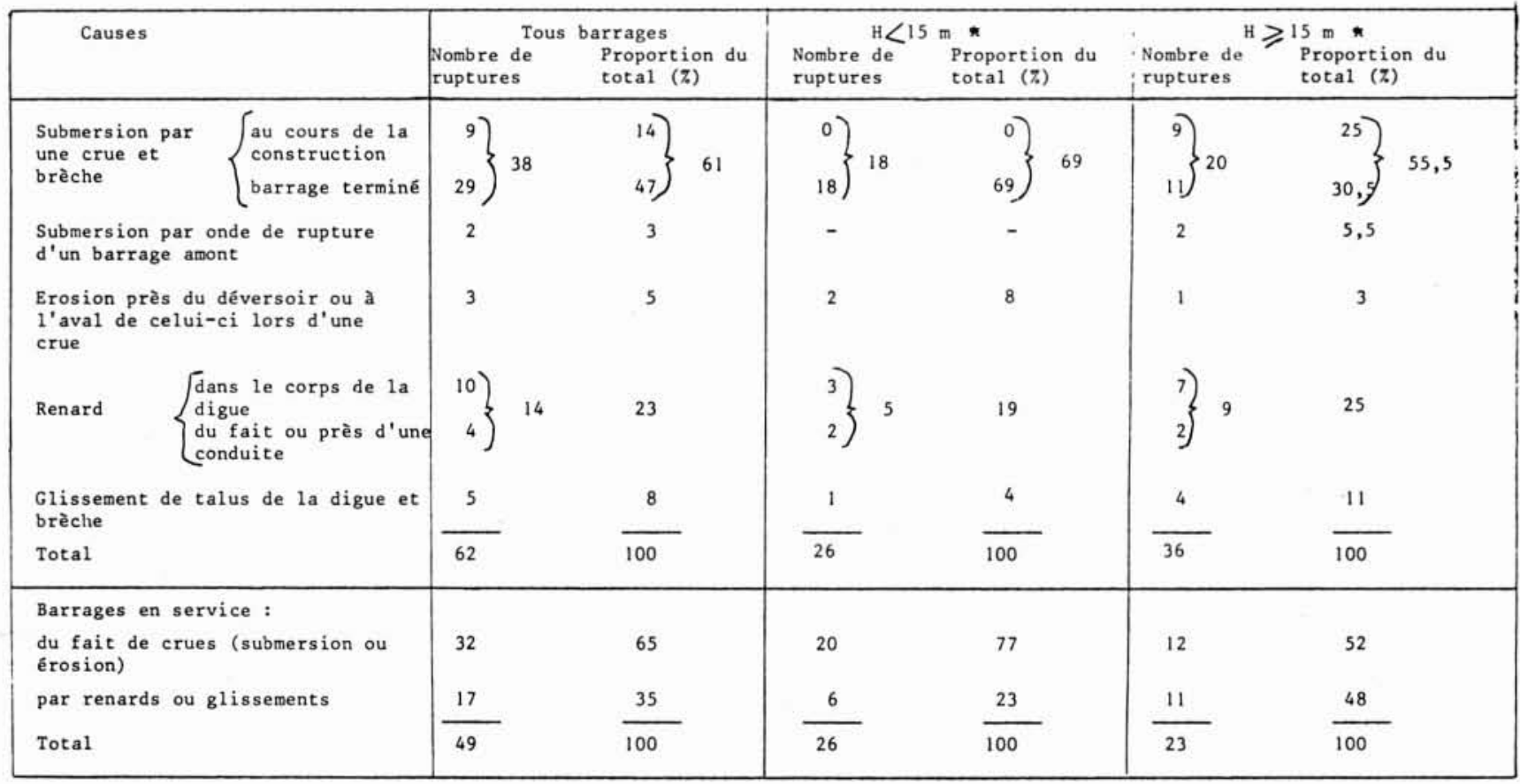

- pour les barrages en construction, hauteur une fois terminés 
Tableau XI. - Estimation du nombre de barrages en service d'au moins $15 \mathrm{~m}$ dans le monde (en 1977).

\begin{tabular}{|c|c|c|c|c|}
\hline Zones géographiques & Digues & Béton ou maçonnerie & Divers & Total \\
\hline USA & 2700 & 700 & 300 & 300 \\
EUROPE & 800 & 1800 & - & 600 \\
RESTE DU MONDE & 5000 & 1400 & - & 600 \\
(JAPON) & $(1200)$ & $(800)$ & $3000)$ \\
\hline TOTAL & 8500 & 3900 & 300 & 12700 \\
\hline
\end{tabular}

Tableau XII. - Nombre de ruptures ayant fait des victimes connues et nombre de victimes connues (1964-1983).

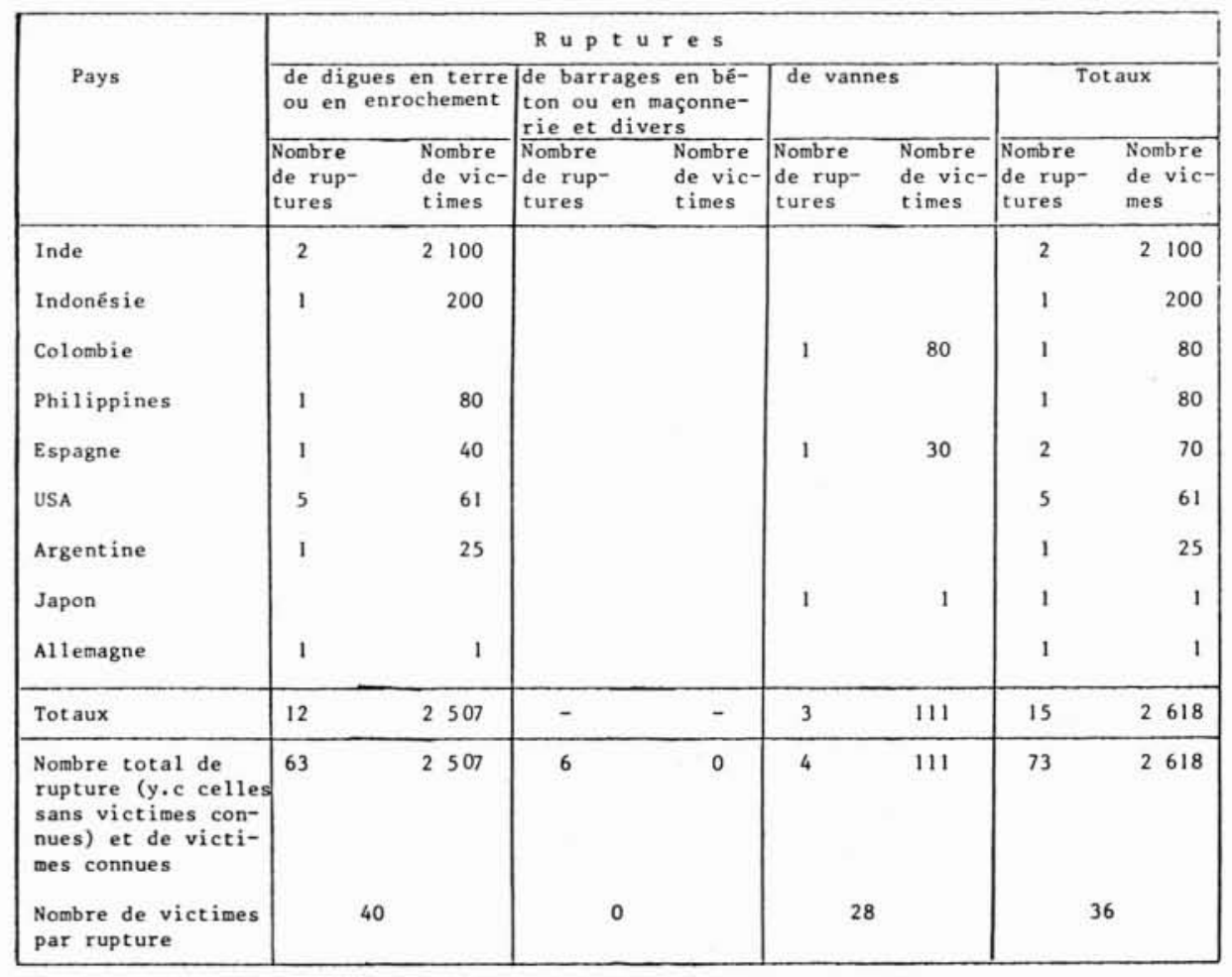




\begin{tabular}{|c|c|c|c|c|c|c|c|c|c|c|c|c|c|c|}
\hline \multirow{2}{*}{ Pays } & \multicolumn{11}{|c|}{ Ruptures } & \\
\hline & \multicolumn{3}{|c|}{\begin{tabular}{|l|} 
de digues en \\
terre ou en- \\
rochements \\
$\mathrm{Nb} \quad \mathrm{N}$
\end{tabular}} & $\begin{array}{l}\text { en bêton } \\
\text { çonnerie } \\
\text { divers } \\
\text { Nb }\end{array}$ & $\begin{array}{l}\text { ma- } \\
\text { e et } \\
\mathrm{N}\end{array}$ & \multicolumn{2}{|c|}{$\begin{array}{l}\text { d'ouvrages } \\
\text { annexes } \\
\text { (vannes) }\end{array}$} & \multicolumn{2}{|c|}{\begin{tabular}{|c|} 
autres (ébou- \\
lement de re- \\
tenue) \\
$\mathrm{Nb}$
\end{tabular}} & \multicolumn{2}{|c|}{$\begin{array}{l}\text { de barrages } \\
\text { de nature } \\
\text { inconnue } \\
\mathrm{Nb} \quad \mathrm{N}\end{array}$} & $\mathrm{Nb}$ & tal & $\mathrm{N}$ \\
\hline Inde & 3 & 3 & 100 & 1 & 1000 & - & - & - & - & - & - & 4 & 4 & 100 \\
\hline Italie & - & & - & 2 & 700 & - & - & 1 & 2600 & - & - & 3 & 3 & 300 \\
\hline USA & 17 & 2 & 5730 & 5 & 541 & - & - & - & - & 3 & 14 & 25 & 3 & 128 \\
\hline All emagne & 1 & & 1 & 2 & 1268 & (2) & - & - & - & - & - & 3 & 1 & 269 \\
\hline Japon & - & & - & - & - & 1 & 1 & - & - & 1 & 1200 & 2 & 1 & 201 \\
\hline Brésil & 1 & 1 & 000 & - & - & - & - & - & - & - & - & 1 & 1 & 000 \\
\hline Espagne & 1 & & 40 & 2 & 752 & 1 & 30 & - & - & - & - & 4 & & 822 \\
\hline France & 1 & & 4 & 2 & 521 & - & - & - & - & - & - & 3 & & 525 \\
\hline Grande Bretagne & 1 & & 238 & 1 & 16 & - & - & - & - & - & - & 2 & & 254 \\
\hline Algérie & - & & - & 2 & 219 & - & - & - & - & - & - & 2 & & 219 \\
\hline Indonésie & 1 & & 200 & - & - & - & - & - & - & - & - & 1 & & 200 \\
\hline URSS & 1 & & 145 & - & - & - & - & - & - & - & - & 1 & & 145 \\
\hline Corée & 1 & & 120 & - & - & - & - & - & - & - & - & 1 & & 120 \\
\hline Chili & 1 & & 100 & - & - & - & - & - & - & - & - & 1 & & 100 \\
\hline Colombie & - & & - & - & - & 1 & 80 & - & - & - & - & 1 & & 80 \\
\hline Philippines & 1 & & 80 & - & - & - & - & - & - & - & - & 1 & & 80 \\
\hline Tchécos lovaquie & 1 & & 65 & - & - & - & - & - & - & - & - & 1 & & 65 \\
\hline Argentine & 1 & & 25 & - & - & - & - & - & - & - & - & 1 & & 25 \\
\hline Canada & 1 & & 1 & - & - & - & - & - & - & - & - & 1 & & 1 \\
\hline Total & 33 & 7 & 692 & 17 & 5017 & 3 & 111 & 1 & 2600 & 4 & 1214 & 58 & 16 & 634 \\
\hline $\begin{array}{l}\text { Ruptures recen- } \\
\text { sées sans } \\
\text { victimes }\end{array}$ & 39 & & 0 & 8 & 0 & 0 & 0 & 0 & 0 & 0 & 0 & 47 & & 0 \\
\hline $\begin{array}{l}\text { Nb de victimes } \\
\text { par rupture } \\
\text { recensée }\end{array}$ & 72 & & 107 & 25 & 200 & 3 & 37 & 1 & 2600 & 4 & 300 & 105 & & 158 \\
\hline
\end{tabular}

Tableau XIII Nombre de ruptures (Nb) ayant fait des victimes connues et nombre de victimes ( $\mathrm{N}$ ) selon le type de rupture et par pays (1800-1983).
(1) dont 2187 pour South Fork, victimes de 1a crue et de 1a rupture

(2) bombardements
Tableau XIV Évolution du nombre de victimes d'accidents de barrages au cours des années depuis 1855 .

\begin{tabular}{|c|c|c|c|}
\hline Année & Nombre & imes & Observations \\
\hline $\begin{array}{l}1855-1860 \\
1860-1865\end{array}$ & $\begin{array}{r}0 \\
238\end{array}$ & 238 & $\begin{array}{l}612 \text { de } 1800 \text { a } 1805 \\
\text { néant de } 1805 \text { a } 1855\end{array}$ \\
\hline $\begin{array}{l}1865-1870 \\
1870-1875\end{array}$ & $\begin{array}{r}1200 \\
143\end{array}$ & 1343 & $\cdot$ \\
\hline $\begin{array}{l}1875-1880 \\
1880-1885\end{array}$ & $\begin{array}{r}0 \\
209\end{array}$ & 209 & \\
\hline $\begin{array}{l}1885-1890 \\
1890-1895\end{array}$ & $\begin{array}{r}2307 \\
134\end{array}$ & 2441 & 2187 pour South Fork, crue incluse \\
\hline $\begin{array}{l}1895-1900 \\
1900-1905\end{array}$ & $\begin{array}{r}115 \\
8\end{array}$ & 123 & \\
\hline $\begin{array}{l}1905-1910 \\
1910-1915\end{array}$ & $\begin{array}{r}0 \\
81\end{array}$ & 81 & \\
\hline $\begin{array}{l}1915-1920 \\
1920-1925\end{array}$ & $\begin{array}{r}1096 \\
600\end{array}$ & 1696 & $\begin{array}{l}1000 \text { a Tigra } \\
600 \text { a Gleno }\end{array}$ \\
\hline $\begin{array}{l}1925-1930 \\
1930-1935\end{array}$ & $\begin{array}{r}467 \\
2\end{array}$ & 469 & \\
\hline $\begin{array}{l}1935-1940 \\
1940=1945\end{array}$ & $\begin{array}{r}101 \\
1268\end{array}$ & 1369 & bombardements \\
\hline $\begin{array}{l}1945-1950 \\
1950-1955\end{array}$ & $\begin{array}{l}0 \\
0\end{array}$ & 0 & \\
\hline $\begin{array}{l}1955-1960 \\
1960-1965\end{array}$ & $\begin{array}{r}565 \\
2 \quad 270\end{array}$ & 2835 & dont Vajont : 2600 morts \\
\hline $\begin{array}{l}1965-1970 \\
1970-1975\end{array}$ & $\begin{array}{r}333 \\
25\end{array}$ & 358 & \\
\hline $\begin{array}{l}1975-1980 \\
1980=1984\end{array}$ & $\begin{array}{r}216 \\
44\end{array}$ & 2260 & 2000 a Macchuu II \\
\hline
\end{tabular}

Sur des périodes de 5 ans

Moyenne 520 soit $100 / a n$ - écart type : 750 Tendance : $R^{2}=0,03$, croissance de 300 à 730 soit 60 par an à 150 par an Sur des périodes de 10 ans Moyenne 1030 soit $100 /$ an - écart type : 1000 Tendance : $\mathrm{R}^{2}=0,08$, croissance de 600 a 1500 soit de 60 par an a 150 par an 


\begin{tabular}{|c|c|c|c|c|c|c|c|c|}
\hline \multirow{2}{*}{$\begin{array}{l}\text { Années de mise } \\
\text { en service }\end{array}$} & \multicolumn{8}{|c|}{ Types de barrage } \\
\hline & $\begin{array}{l}\text { Digues en } \\
\text { terre ou } \\
\text { en terre et } \\
\text { enrochement }\end{array}$ & $\begin{array}{l}\text { Digues } \\
\text { en } \\
\text { enroche- } \\
\text { ment }\end{array}$ & $\begin{array}{l}\text { Poids (y. } \\
\text { c. barra- } \\
\text { ges mobi- } \\
\text { les }\end{array}$ & $\begin{array}{l}\text { Poids- } \\
\text { voûtes }\end{array}$ & Voûtes & $\begin{array}{l}\text { Voûtes } \\
\text { multi- } \\
\text { ples }\end{array}$ & $\begin{array}{l}\text { a con- } \\
\text { trefort }\end{array}$ & Total \\
\hline $1600-1699$ & 1 & & & & & & & 1 \\
\hline $1700-1799$ & & & 2 & & & & & 2 \\
\hline $1800-1849$ & 4 & & 5 & & & & & 9 \\
\hline $1850-1899$ & 6 & 2 & 14 & & 1 & & & 23 \\
\hline $1900-1909$ & 4 & & 12 & & & & & 16 \\
\hline $1910-1919$ & & & 7 & & 1 & 1 & & 9 \\
\hline $1920-1929$ & & & 24 & & 2 & 1 & & 27 \\
\hline $1930-1939$ & 2 & 3 & 22 & & 4 & 3 & & 34 \\
\hline $1940-1949$ & 1 & 3 & 14 & 1 & 13 & 2 & 2 & 36 \\
\hline $1950-1954$ & 1 & 4 & 12 & 2 & 24 & 2 & & 45 \\
\hline $1955-1959$ & 2 & 1 & 10 & & 13 & 3 & 1 & 30 \\
\hline $1960-1964$ & 11 & 2 & 13 & & 11 & & 4 & 41 \\
\hline $1965-1969$ & 11 & 3 & 18 & 1 & 7 & 1 & 1 & 42 \\
\hline $1970-1974$ & 15 & 9 & 15 & & 3 & 2 & 3 & 47 \\
\hline $1975-1979$ & 22 & 6 & 10 & & 3 & 1 & 3 & 45 \\
\hline $1980-1984(1)$ & 21 & 7 & 9 & & 2 & 1 & 3 & 43 \\
\hline Total & $\begin{array}{l}101 \\
\text { digues : } 14\end{array}$ & $41^{40}$ & $\begin{array}{c}187 \\
\text { en béton }\end{array}$ & $\begin{array}{c}4 \\
\text { et maçon }\end{array}$ & $\begin{array}{l}84 \\
\text { rie : } 30\end{array}$ & 17 & 17 & 450 \\
\hline
\end{tabular}

(1) selon prévision de mise en service après 1982.

Tableau XV

Barrages en service en France, selon la définition CIGB (répertoire à jour en 1982).

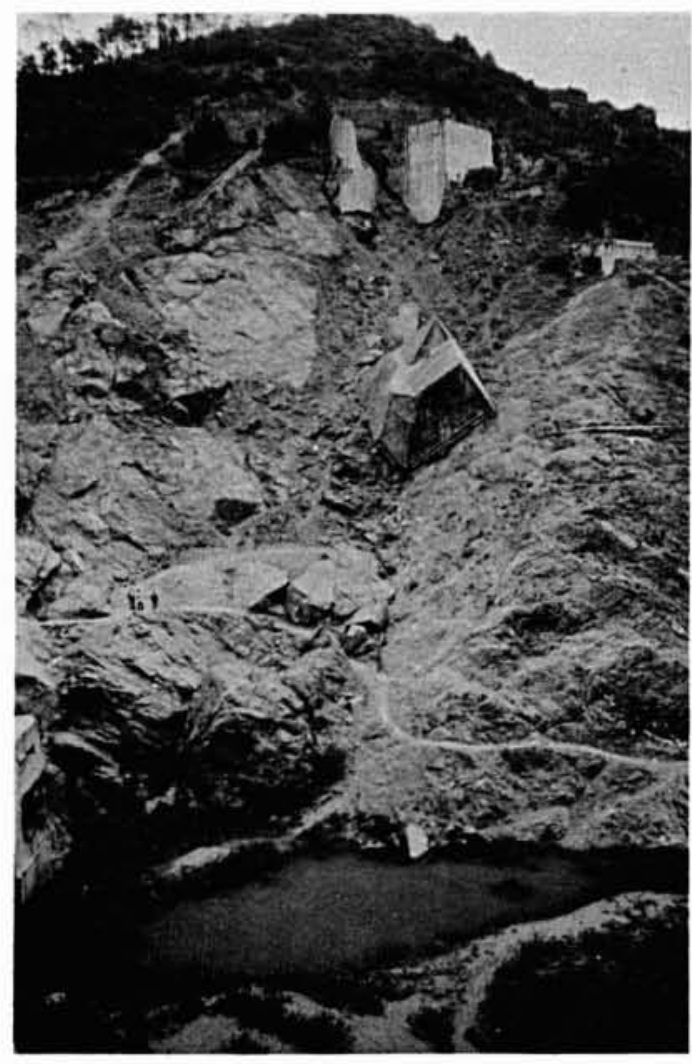

Malpasset

Le "diedre " rive gauche $V u$ du versant R.D. 
ANNEXE I

Distribution des accidents selon le nombre de victimes

(1964-1983)

On distingue sur la figure $I$, deux populations, l'une d'allure log-normale, l'autre d'allure normale. La première n'a pas été estimée, le nombre de classes étant trop faible. L'estimation de la population d'allure normale donne les résultats suivants :

Nombre $=11-$ Moyenne $: 4-$ Ecart type $: 1,57$. Le test de Shapiro-Wilk est acceptable.

Cette moyenne correspond à $N=55$. A $m-\sigma$ correspond $N=12$ et à $m+\sigma, N=270$. Aux fréquences faibles de dépassement correspondent les valeurs suivantes :

$$
\begin{array}{lllll}
F & 0,9 & 0,99 & 0,999 & 0,9999 \\
N & 420 & 2150 & 7100 & 19000
\end{array}
$$

Si l'on rapporte ces fréquences à la population totale d'accidents, environ 6 fois plus nombreuse environ que la population normale, on trouve :

$\begin{array}{lllll}F & 0,9 & 0,99 & 0,999 & 0,9999\end{array}$

Fréquence de dépassement

$$
\begin{array}{ccccc} 
& 10 \% & 1 \% & 1 / 1000 & 1 / 10000 \\
N & 38 & 650 & 2900 & 9000
\end{array}
$$

Pour les seuls barrages d'au moins $15 \mathrm{~m}$, la population totale d'accidents n'est que 4 fois plus nombreuse que la population normale et les fréquences deviennent :

$$
\begin{array}{lllll}
F & 0,9 & 0,99 & 0,999 & 0,9999
\end{array}
$$

Fréquence de dépassement

$\begin{array}{lcccc} & 10 \% & 1 \% & 1 / 1000 & 1 / 10000 \\ N & 100 & 950 & 3900 & 11000\end{array}$

Note $: \mathrm{Nb}:$ nombre d'accidents

$\mathrm{N}$ : nombre de victimes par accident

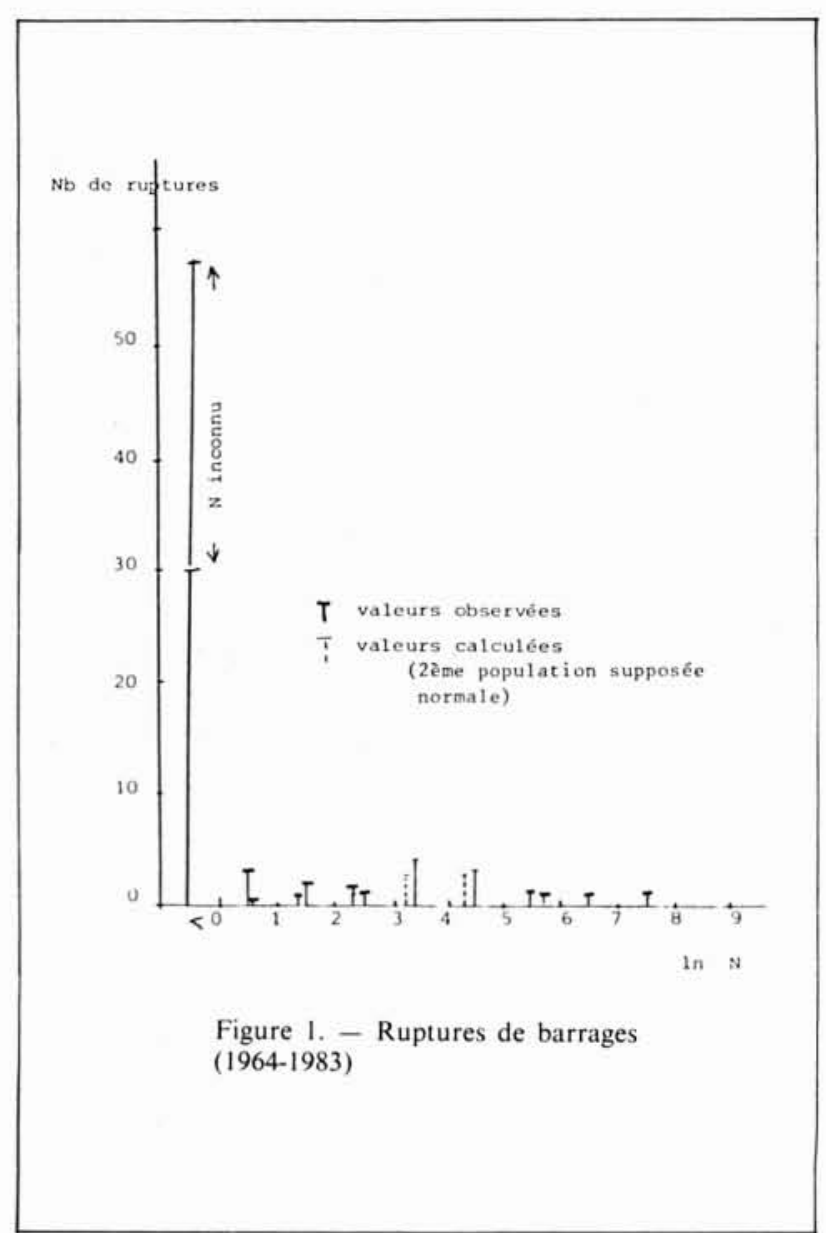

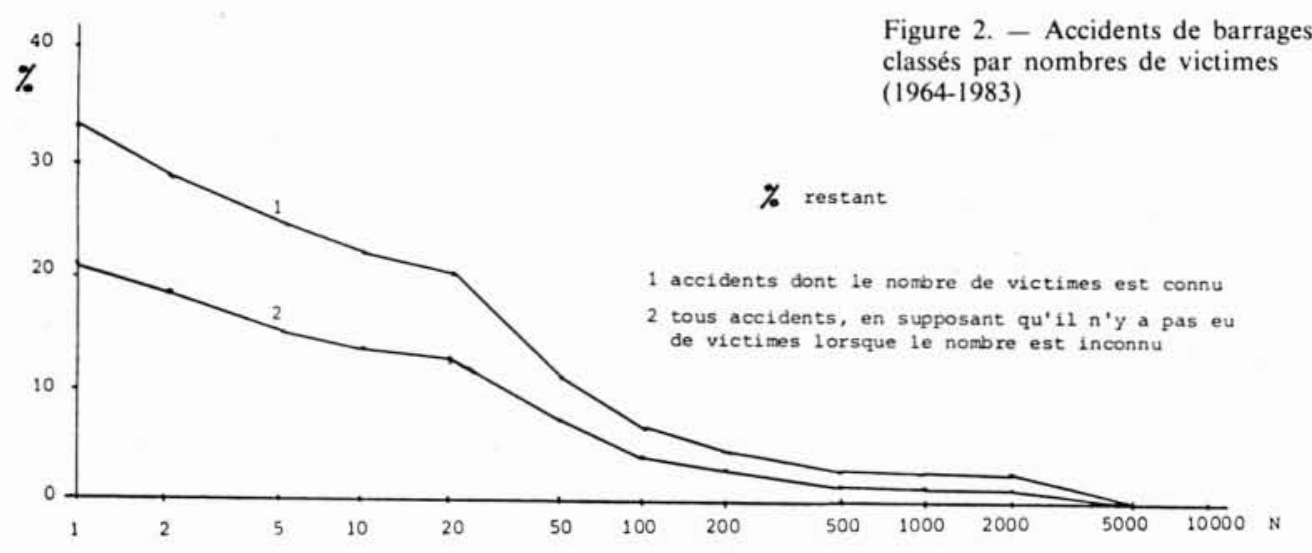


ANNEXE II

Distribution des accidents selon le nombre de victimes

(1800-1983)

\section{A - Tous barrages}

On distingue, sur la figure 3, deux populations, l'une d'allure log-normale, l'autre d'allure normale. Les estimations de ces deux populations donnent les résultats suivants :

Population log-normale

$$
N B=68 \quad M=0,0524 \quad \sigma=0,55
$$

Le test du $X^{2}$ est médiocre $\left(X^{2}=10,24 ; \alpha\right.$ de l'ordre de $0,001)$.

\section{Population normale}

$$
N B=36 m=5,1 \quad \sigma=1,55
$$

Le test du $X^{2}$ est correct $\left(X^{2}=0,6 ; \alpha=0,90\right)$

Cette moyenne correspond à $N=164$. A $m-\sigma$ correspond $N=35$, à $m+\sigma . N=770$. Aux fréquences faibles de dépassement correspondent les valeurs suivantes :

$$
\begin{array}{ccccc}
F & 0,9 & 0,99 & 0,999 & 0,9999 \\
N & 1200 & 5800 & 19000 & 50000
\end{array}
$$

$\mathrm{Si}$ l'on rapporte ces fréquences à la population totale d'accidents, environ 4 fois plus nombreuse que la population normale, on trouve :

Fréquence de dépassement

$$
\begin{array}{ccccc}
F & 0,9 & 0,99 & 0,999 & 0,9999 \\
& 10 \% & 1 \% & 1 / 1000 & 1 / 10000 \\
N & 240 & 2500 & 10000 & 30000
\end{array}
$$

Note: $\mathrm{Nb}$ : nombre d'accidents

$$
\mathrm{N} \text { : nombre de victimes par accident }
$$

\section{B - Digues}

La population log-normale $(\mathrm{Nb}=51)$ n'a pas été estimée. $\mathrm{La}$ population normale $(\mathrm{Nb}=21)$ a pour moyenne $m=4,6(N=100)$ et écart type $\sigma=1,69$. Le test du $X^{2}$ $\left(X^{2}=5,6\right)$ et celui de Shapiro-Wilk sont médiocres. A $m$ - $\sigma$ correspond $N=19$ et à $m+\sigma, N=550$. Les fréquences extrêmes, pour la seule population normale dont il est difficile de dire la part dans la population totale, sont les suivantes :

$$
\begin{array}{ccccc}
F & 0,9 & 0,99 & 0,999 & 0,9999 \\
N & 900 & 5200 & 19000 & 54000
\end{array}
$$

Ces valeurs sont proches de celles obtenues pour tous les barrages mais elles ne leur sont pas directement comparables, certaines ruptures ayant été exclues (vannes, éboulements de rives, nature de barrage inconnue).

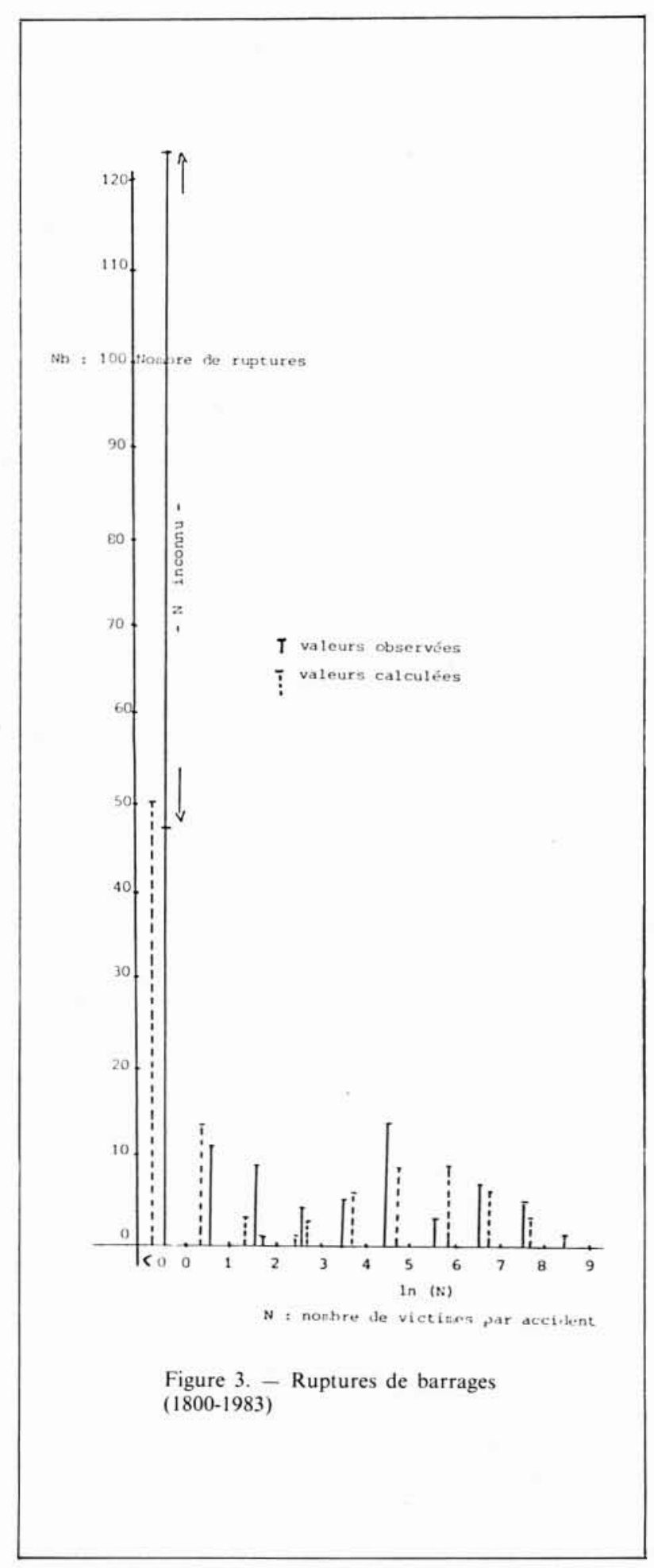


(Annexe II - suite)

\section{C - Barrages en béton et maçonnerie}

La population est plus réduite. La population log-normale $(\mathrm{Nb}=11)$ n'a pas été estimée. La population normale $(\mathrm{Nb}$ =14) a pour moyenne $m=5(N=150)$ et écart type $\sigma=1,67$. A $m-\sigma$ correspond $N=28$ et à $m+\sigma, N$ $=780$. Le test de Shapiro Wilk est médiocre. Les fréquen- ces extrêmes pour la seule population normale, sont les suivantes :

$\begin{array}{ccccc}F & 0,9 & 0,99 & 0,999 & 0,9999 \\ N & 1250 & 7000 & 25000 & 70000\end{array}$

Cela donne des valeurs plus élevées d'environ $1 / 3$ que les valeurs obtenues pour les digues. Les valeurs correspondantes à la médiane, à $m-\sigma$ et à $m+\sigma$ sont environ $50 \%$ plus fortes.

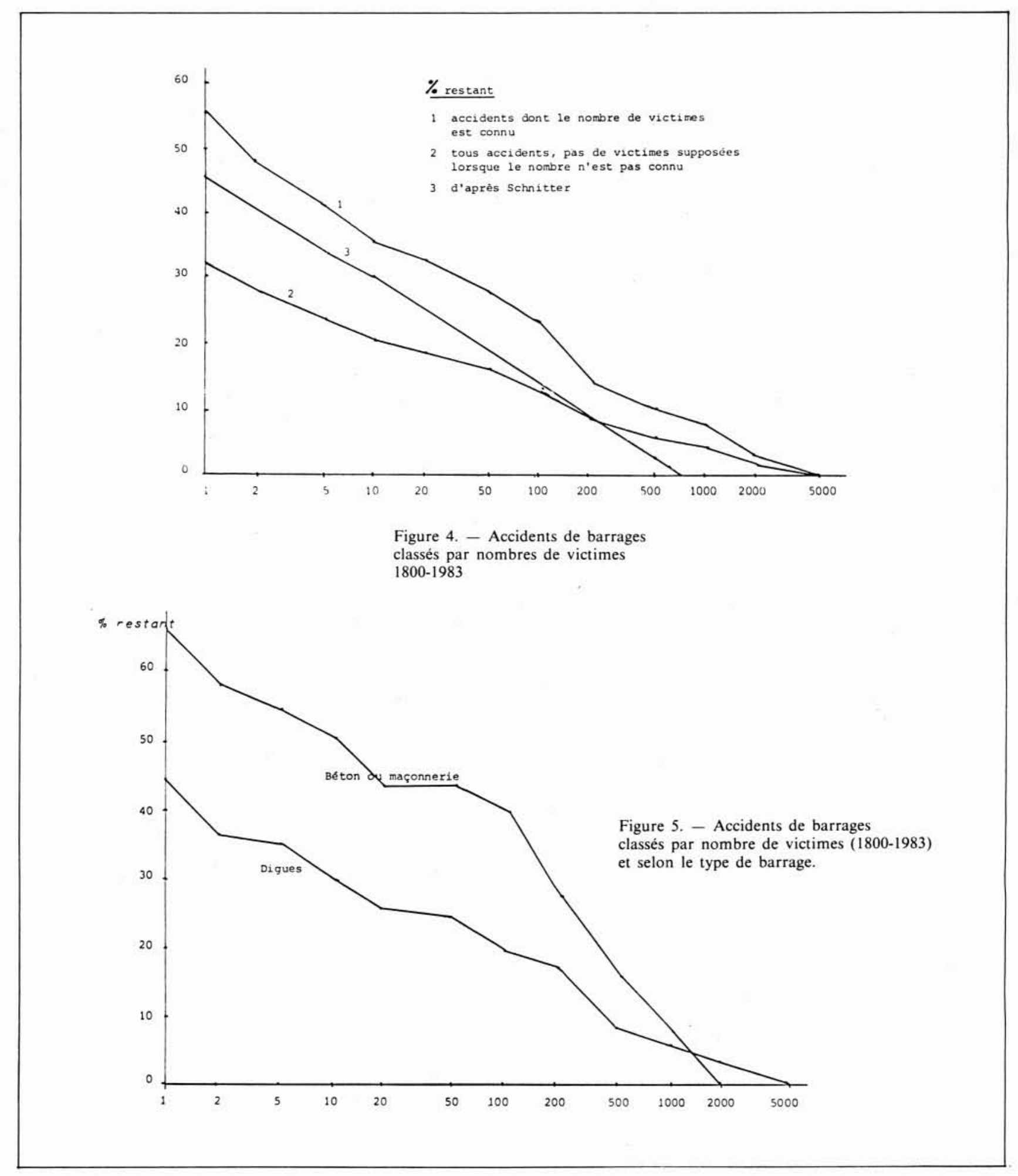

